\title{
Los diseños de índices de divulgación de información financiera en Internet. Una revisión sistemática de la literatura*
}

\author{
The designs of financial information disclosure indices on the Internet. A systematic literature review \\ Os designs dos índices de divulgação de informações financeiras na Internet. Uma revisão sistemática da literatura
}

Carlos Augusto Rincón-Soto ${ }^{\mathrm{a}}$

Universidad del Valle, Colombia

carlos.augusto.rincon@correounivalle.edu.co

ORCID: https://orcid.org/0000-0003-4710-5558

DOI: https://doi.org/10.11144/Javeriana.cao34.didifi

Mauricio Gómez-Villegas

Universidad Nacional de Colombia, Colombia

ORCID: https://orcid.org/0000-0001-9043-6358

Recibido: 23 Septiembre 2020

Aceptado: 27 Septiembre 2021

Yaismir Adriana Rivera-Arrubla

Universidad del Valle, Colombia

ORCID: https://orcid.org/0000-0002-8538-4925

Publicado: 30 Diciembre 2021

\section{Resumen:}

El propósito de este artículo es sintetizar y describir los diseños de los índices utilizados en la investigación sobre la divulgación de Información Financiera en Internet (IFI). Se hizo una revisión sistemática de la literatura y se analizaron 46 artículos de investigación con enfoque cuantitativo-explicativo, dedicados a estudiar la divulgación de IFI y que publican el diseño del índice usado. Los resultados permiten sistematizar los diseños más comunes en los índices, dimensiones, cantidad de ítems, así como las teorías, metodologías, variables determinantes y los hallazgos más significativos en la investigación académica. Se concluye que la heterogeneidad en la construcción y en las características de estos índices, es producto de la evolución en la que se encuentra dicho campo de investigación.

Códigos JEL: D89, G00

Palabras clave: Revisión sistemática de la literatura, sitios web corporativos, transparencia, índices de divulgación.

Abstract:

The purpose of this article is to synthesise and describe the designs of indices used in research related to Internet Financial Reporting (IFR). A systematic literature review analysed 46 research articles with a quantitative-explanatory approach; these articles were all dedicated to studying IFR and included information about the index design used. The results allow for the systematisation of the most common designs by the indices, dimensions, and number of items, as well as the theories, methodologies, determining variables, and the most significant findings in academic research. It is concluded that the heterogeneity in the construction and characteristics of these indices is the product of the evolution of this field of research.

JEL Codes: D89, G00

Keywords: Systematic literature review, corporate websites, Transparency, disclosure index.

\section{Resumo:}

O objetivo deste artigo é sintetizar e descrever os designs dos índices utilizados nas pesquisas sobre divulgação de Informações Financeiras na Internet (IFI). Foi realizada uma revisão sistemática da literatura e analisados 46 artigos de pesquisa com abordagem quantitativo-explicativa, que se dedicam a estudar a divulgação de IFI e que publicam o design do índice utilizado. Os resultados permitem sistematizar os designs mais comuns nos índices, dimensões, número de itens, bem como nas teorias, metodologias, variáveis determinantes e achados mais significativos na pesquisa acadêmica. Conclui-se que a heterogeneidade na construção e nas características desses índices é produto da evolução em que este campo de pesquisa se encontra.

Códigos JEL: D89, G00

Palavras-chave: Revisão sistemática da literatura, sites corporativos, transparência, índices de divulgação.

Notas de autor

\footnotetext{
${ }^{a}$ Autor de correspondencia. E-mail: carlos.augusto.rincon@correounivalle.edu.co
} 


\section{Introducción}

Las tecnologías de la información y la comunicación (TIC), principalmente Internet, han ampliado la posibilidad de divulgación y transparencia corporativa, lo que ha permitido que las empresas publiquen información financiera y no financiera en sus páginas web, con intención de ampliar su relación con los accionistas, los clientes y otros stakeholders (Ali Khan \& Ismael, 2012a).

El Internet mejora el acceso a la información, permite mostrar datos en diversos formatos (en video, audio, presentación, hojas de cálculo, entre otros), es una herramienta de bajo costo, aumenta la oportunidad y reduce la asimetría de la información (Leão, João, \& Castelló, 2018). Estas ventajas determinaron los cambios en la presentación y divulgación de la información financiera y no financiera de las empresas en todo el mundo (Mokhtar, 2017).

La divulgación de información financiera a través de Internet se interpreta por los stakeholders como una búsqueda de transparencia empresarial (Montesinos, 2009). Este tipo de práctica se ha extendido más rápidamente en algunos sectores, regiones, industrias y grupos empresariales, que en otros.

El estudio del uso de Internet para la divulgación de información financiera es importante para académicos y reguladores contables, quienes buscan comprender y explicar la evolución de esta tendencia y sus impactos en la transparencia corporativa (Ali Khan \& Ismael, 2012a; Aranguren \& Maldonado, 2015). Asimismo, es importante para gobiernos y organizaciones de control y vigilancia de los sectores empresariales, quienes reclaman de las empresas más acceso a la información que interesa al público (Mokhtar, 2017). La información corporativa divulgada en Internet reta a los preparadores y evaluadores de la información -auditores-, debido a los cambios de formato, canal y a las nuevas exigencias de divulgación, construcción de confianza y aseguramiento de la información (Fisher \& Naylor, 2016). En este contexto, diversos actores plantean la necesidad de estándares que guíen tanto la divulgación de información financiera electrónica, como las exigencias legales por parte de los gobiernos (Xiang \& Birt, 2021).

Aunque la producción académica sobre IFI es significativa, aún es un campo de estudio en evolución (Rodríguez, Alcaide, \& López, 2013; Xiao, Yang, \& Chow, 2004). En general, la investigación de IFI se desarrolla bajo metodologías descriptivas y explicativas (Ali Khan \& Ismael, 2012a; Maldonado-García, Escobar-Váquiro, \& Marín, 2012), que recurren al uso de índices con el propósito de obtener la información sobre las prácticas en esta materia. Dichas prácticas varían según la especificidad del objetivo y del contexto de estudio, por lo que existe la necesidad de sistematizar y comprender las dimensiones contextuales, sociales y culturales de la utilización de Internet para ampliar la transparencia corporativa.

Para Xiao et al. (2004) y Mokhtar (2017), el incremento del uso de Información Financiera en Internet (IFI), plantea preguntas sobre las variables con incidencia y los determinantes de dicha divulgación, pero aún no hay consenso sobre las características y condiciones que explican la divulgación diferenciada en sectores e industrias, así como sus impactos reales en la transparencia.

Este trabajo busca sintetizar y describir la estructura del diseño de los índices utilizados en la investigación sobre la divulgación de IFI, identificando las características, dimensiones, ítems, teorías, metodologías y los hallazgos más significativos que explican la divulgación de reportes financieros en Internet por parte de las empresas. Este trabajo busca contribuir a la literatura, realizando una revisión sistemática que identifica la divulgación de reportes financieros por medio del índice de divulgación de IFI.

Analizar la forma en que se viene investigando y midiendo la divulgación IFI por medio de diversos índices, ayudará a caracterizar los instrumentos y los avances en este campo de la información empresarial. 


\section{Antecedentes de la investigación}

En la literatura académica se suele definir la IFI a partir de sus contenidos y de su papel en el análisis de los comportamientos de divulgación del informe anual financiero y corporativo publicados en la web. Pina, Torres \& Royo (2010) y Qasim \& Al Barghouthi (2017) definen la IFI como la divulgación de elementos relacionados con el informe anual en las páginas web corporativas. Para Lee \& Joseph (2013) este concepto se refiere a la divulgación obligatoria y voluntaria de información financiera y de desempeño de alta calidad en el sitio web de la organización. Para Yusuf (2013) el estudio sobre IFI consiste en la revisión y análisis sobre el comportamiento de las empresas que tienen un sitio web y presentan de manera on-line sus informes anuales y otra información financiera. Este tipo de estudios analizan la calidad de lo divulgado y la transparencia de las empresas, pues se plantea que las buenas prácticas de divulgación de IFI disminuyen la asimetría de la información, mejoran la imagen corporativa y aumentan la confianza de los stakeholders (FuertesCallén, Cuellar-Fernández, \& Pelayo-Velázquez, 2014; Gandía, 2008), al tiempo que contribuyen a mitigar la corrupción (Leão et al., 2018). Otros estudios estrechamente relacionados son aquellos sobre Informes Corporativos en Internet, los cuales incluyen datos de IFI y otra información sobre inversores, gobierno corporativo, responsabilidad social o ambiental (Bonsón \& Escobar, 2004).

Las investigaciones en IFI se pueden categorizar en dos corrientes: (i) la que tiene por objetivo describir el uso de IFI por parte de las empresas para comunicar y ofrecer confianza a los stakeholders, y (ii) la que mide el nivel de divulgación IFI y sus determinantes (Mokhtar, 2017). Las investigaciones descriptivas en IFI surgieron en la década de 1990 (Ashbaugh, Johnstone, \& Warfield, 1999), las cuales comparaban las diferencias de las prácticas de divulgación IFI en contextos determinados, generalmente, empresas que se encuentran en el mercado bursátil, comparación entre empresas de distintos países, bolsas de valores e industrias. En la década de 2000 se manifiestó la tendencia de estudios explicativos, cuestión que se ha intensificado en la segunda década del siglo XXI (Pinto \& Ng Picoto, 2016). Esta tendencia tiene como propósito entregar evidencia empírica sobre los determinantes que explican las prácticas y los niveles de divulgación de IFI (Ali Khan \& Ismael, 2012b, 2012a).

Ali Khan \& Ismael (2011) revisaron 32 artículos publicados entre 1999 a 2010 relacionados con IFI y determinaron la cantidad de dimensiones e ítems que se utilizan para el diseño de los índices. Los autores plantean que, tradicionalmente, las estructuras de análisis suelen dividirse en dos dimensiones: de contenido y de presentación. Posteriormente, Ali Khan \& Ismael (2012b) evaluaron en la dimensión de contenido 97 ítems y en la dimensión de presentación 58 ítems, con 68 expertos de contabilidad y finanzas involucrados en IFI en Malasia. Utilizaron una encuesta con una escala de likert de 1 a 5, siendo 5 la opinión de mayor relación significativa. En los resultados de la dimensión de contenido se obtuvo una evaluación superior a 3,5 para 67 ítems. Este estudio permite visualizar el nivel de importancia que le dan los expertos a cada ítem para evaluar la calidad de divulgación IFI.

Ali Khan \& Ismael (2012a) revisó 29 artículos de la corriente explicativa, publicados entre 1996 y 2008, donde examinan las variables independientes utilizadas, concluyendo que los investigadores trabajan distintos diseños de índices en dimensiones y que esto genera inconsistencias para integrar el conocimiento existente. Asimismo, establecen que las variables independientes más investigadas son tamaño de la empresa, rentabilidad y apalancamiento; y la menos testeada es la puntualidad.

Rodríguez, Alcaide \& López (2013) realizaron un metaanálisis de 39 artículos sobre la relación entre los factores ambientales (tamaño, riqueza de población) y los factores institucionales (subsidios intergubernamentales, situación financiera de las organizaciones, competencia política), con respecto a la estrategia de transparencia de la información y la accountability pública en la divulgación de información en Internet, respaldados por la teoría de agencia, la teoría neoinstitucional y la teoría de la legitimidad. La investigación muestra que los trabajos tienen una relación significativa de los factores seleccionados y que los resultados son altamente influenciados por el contexto. 
Sharma (2013) realizó un estudio sobre los referentes teóricos utilizados en los trabajos de investigación sobre divulgación de información financiera en estudios descriptivos y explicativos, donde plantea que la calidad de la teoría depende de su capacidad para explicar las variables relacionadas. Concluye que las teorías más utilizadas en este tipo de investigación son la teoría de la agencia, la teoría de la asimetría de la información, la teoría de la señalización, la teoría de las partes interesadas y la teoría institucional.

Mokthar (2017) realizó un meta-análisis para examinar las variables independientes que se han utilizado en los trabajos sobre divulgación de IFI para explicar sus resultados, a partir de la revisión de 59 artículos de investigación. Las variables estudiadas presentan una asociación positiva y significativa con el tamaño de la empresa, rentabilidad, apalancamiento, el tipo de auditor y los niveles de divulgación IFI. También se identifican como referentes conceptuales: la teoría de la agencia, la teoría de la señalización, la hipótesis del costo político y la teoría de la difusión de la innovación.

Algunos autores plantean que no hay una adecuada regulación para definir y controlar la información transmitida en las páginas web corporativas, lo que conduce a diferencias en los contenidos de IFI, dado que estos están supeditados al interés de los directivos y a las presiones institucionales (Ali Khan \& Ismael, 2012a). Asimismo, plantean que a pesar de la existencia de una cantidad de estudios interesantes, todavía falta llegar a consensos y a una robustez temática. En esta línea, varios autores recomiendan hacer más investigación para llenar los vacíos existentes con respecto a la divulgación IFI (Mokhtar, 2017; Pinto \& Ng Picoto, 2016).

\section{Metodología}

La revisión sistemática de la literatura ha sido adoptada como una metodología empírica válida, empleada en diferentes campos de investigación para sintetizar y analizar de manera rigurosa los resultados de las investigaciones, buscando el consenso y las diferencias existentes en un campo o un tema de investigación específico (Siddaway, Wood, \& Hedges, 2019; Stechemesser \& Guenther, 2012).

Ali Khan e Ismael (2010, 2012a, 2012b) hicieron revisiones de literatura sobre IFI, de estudios tanto descriptivos como explicativos entre las décadas de 1990 y 2010. La tendencia de la segunda década del siglo XXI está relacionada con corrientes explicativas. Por lo tanto, este artículo busca bajo una revisión sistemática de la literatura, identificar las características y los resultados en el diseño de los índices para las investigaciones explicativas sobre IFI, ampliando el margen de tiempo de los estudios previos, incluyendo artículos publicados en el período 2000-2021.

Para la revisión sistemática se siguieron los pasos recomendados por Stechemesser \& Guenther (2012), a saber: seleccionar las preguntas de investigación, las funciones de búsqueda, las bases de datos y establecer los criterios para inclusión y exclusión de la literatura. La búsqueda se realizó por la recomendación encontrada en Ali Khan \& Ismael (2011) sobre los distintos nombres bajo los cuales se podrían encontrar las investigaciones en IFI $^{1}$. Se utilizaron las bases de datos Scopus y Web of science, igualmente, se recurrió a Scielo y Redalyc, con la intención de no descartar investigaciones locales de gran importancia a la hora de observar los contextos de este tipo de estudios.

Se encontraron 102 artículos publicados en inglés y español, de los cuales se descartaron 36 porque no publicaban el índice propuesto, requisito necesario para el análisis de este estudio, pues se pretende conocer cuáles son las dimensiones e ítems que presenta el diseño del índice. Se descartaron 15 trabajos que no eran explicativos, dado que se requiere analizar cuáles son las variables independientes, así como cinco artículos que no se relacionaban esencialmente con IFI, analizando finalmente 46 artículos. Además, se utilizó el programa VOSviewer para visualizar las redes bibliométricas de los artículos clasificados (Van \& Waltman, 2010). 


\section{Resultados}

De los artículos identificados y seleccionados (ver tabla 1), la primera corriente de publicación correspondiente a la década de 2010 se focaliza en países desarrollados, pero para la segunda década del siglo XXI se observa un aumento de estudios en países emergentes ubicados en Latinoamérica, África y Asia. Estos estudios encuentran que los países desarrollados tienen mejores prácticas de divulgación en Internet que los países emergentes (Alali \& Romero, 2012), dejando entrever que el contexto y los elementos culturales son variables importantes en los resultados de la IFI.

Como se aprecia en la tabla 1, la mayoría de los trabajos fueron realizados en empresas cotizadas, lo que implica que se esperaría un mayor nivel de divulgación y transparencia, por tratarse de empresas públicas. En menor cantidad se encuentran investigaciones en empresas financieras y del sector bancario, municipios o localidades y escasamente una en empresas no cotizadas. La investigación con mayor número de observaciones (1.806) se focaliza en municipios, seguida de un estudio de empresas cotizadas en varios continentes con 882 observaciones, cantidad que está por fuera del promedio de observaciones de los artículos sobre este tema y donde el menor número de observaciones fue de 33. El promedio de observaciones es de 203. Por lo general, las observaciones corresponden al análisis de un solo año, mientras cuatro investigaciones estudian más de un período. Una de las dificultades de los estudios longitudinales es la observación o elaboración de los índices de divulgación IFI en varios periodos.

La tabla 1 también permite identificar a los autores más citados en Google Scholar. En la línea de IFI del listado de estudio se encuentra en primer lugar Xiao et al. (2004), seguido por Kelton \& Yang (2008). En Informes Corporativos en Internet se encuentra Marston \& Polei (2004), Akhtaruddin (2005) y Mohd \& Weetman (2006). Los trabajos más destacados en Latinoamérica son Alali \& Romero (2012) y Fuertes-Callén et al. (2014).

Para Xiao et al. (2004) los resultados de la divulgación IFI están sujetos a influencias sistemáticas y no al azar, lo que hace importante los estudios explicativos. Asimismo, los investigadores confluyen en que los estudios en IFI e Informes Corporativos en Internet se han vuelto interesantes, dado que el mejoramiento de la divulgación de información financiera y no financiera en Internet está relacionado con el aumento de las posibilidades de inversión y la llegada de nuevos inversores extranjeros (Dolinšek \& Lutar-Skerbinjek, 2018). 
TABLA 1

Artículos con contenido de IFI que incluyen el diseño de un índice, por año de publicación

\begin{tabular}{|c|c|c|c|c|c|c|c|c|c|c|}
\hline \# & Autores & Tipo & $\begin{array}{l}\text { Tipo de } \\
\text { empresas }\end{array}$ & $\begin{array}{l}\text { Paises o } \\
\text { regiones }\end{array}$ & obs" & $\begin{array}{l}\text { Año de } \\
\text { estudio }\end{array}$ & Dimensiones & items & $\begin{array}{c}\text { Variables } \\
\text { independien- } \\
\text { tes }\end{array}$ & $\mathrm{Cit}^{2 /}$ \\
\hline 1 & $\begin{array}{l}\text { Xiao et al. } \\
(2004)\end{array}$ & $|F|$ & Coitzadas & China & 300 & 2002 & $\begin{array}{c}\text { Contenido, } \\
\text { presentiacón }\end{array}$ & 82 & 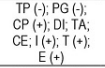 & 794 \\
\hline 2 & $\begin{array}{l}\text { Marston \& Polei } \\
\text { (2004) }\end{array}$ & ICI & Cotizadas & Alemania & 50 & $\begin{array}{l}2000 y \\
2003\end{array}$ & $\begin{array}{l}\text { Contenido, } \\
\text { presentacóón }\end{array}$ & 62 & $\begin{array}{l}T(+) ; C E(+) ; \\
R S ; R ; T\end{array}$ & 650 \\
\hline 3 & $\begin{array}{l}\text { Bonsón \& } \\
\text { Escobar (2004) }\end{array}$ & $\mathrm{ICI}$ & Cotizadas & $\begin{array}{l}\text { Estatos Uniosios. } \\
\text { Euroopadel Este- } \\
\text { Isandiay la } \\
\text { Union Europea }\end{array}$ & 882 & 2002 & items & 23 & I. LG; $T(+)$ & 6 \\
\hline 4 & $\begin{array}{l}\text { Akhtaruddin } \\
\text { (2005) }\end{array}$ & $\mathrm{ICI}$ & Cotizadas & Bangladesh & 94 & 1999 & $\begin{array}{l}\text { Resumen histórico, } \\
\text { elementios del } \\
\text { balance, estadode } \\
\text { resultadosos, politicas } \\
\text { de contabilitad, } \\
\text { Informe del director }\end{array}$ & 160 & $\begin{array}{l}\text { A: Et:C; } \mathrm{V}(+) ; \\
\operatorname{ROEEE}(+) ; \\
\operatorname{RoV}(+)\end{array}$ & 533 \\
\hline 5 & $\begin{array}{l}\text { Aksu \& Kosedag } \\
(2006)\end{array}$ & ICI & Cotizadas & Estambul & 52 & 2003 & $\begin{array}{c}\begin{array}{c}\text { Propiedad, } \\
\text { informacion } \\
\text { financier, untay y } \\
\text { gerencia }\end{array} \\
\end{array}$ & 106 & $T(+) ; V L(-) ; R F ;$ & 288 \\
\hline 6 & $\begin{array}{l}\text { Mohd \& } \\
\text { Weetman (2006) }\end{array}$ & ICI & Cotizadas & Malasia & 100 & 2001 & 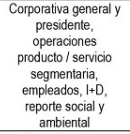 & 53 & $\begin{array}{c}\mathrm{CP}(+) ; \mathrm{NA} ; \\
\mathrm{PD}(+) ; \mathrm{PG}(+) ; \\
\mathrm{DF}(+) ; \mathrm{DI}(+) ; \\
\mathrm{VM}(+) ; \mathrm{CP}(+) ; \\
\mathrm{NE}: \mathrm{R} ; \mathrm{E}\end{array}$ & 518 \\
\hline 7 & $\begin{array}{l}\text { Siram \& } \\
\text { Laksmana } \\
\text { (2006) }\end{array}$ & $\mathrm{ICl}$ & $\begin{array}{l}\text { Alta } \\
\text { tecnologia }\end{array}$ & Fortune-1000 & 212 & 2002 & $\begin{array}{l}\text { Financieros y yo } \\
\text { financieros, análisis } \\
\text { deg gestión, } \\
\text { rrospectiva, } \\
\text { empleades objetivos } \\
\text { yestrategias }\end{array}$ & 26 & $\begin{array}{l}T(+) ; 1(+) ; i \\
R(+) ; \operatorname{CE}(+) ; \\
\operatorname{PEV}(+)\end{array}$ & 46 \\
\hline 8 & $\begin{array}{l}\text { Serrano, } \\
\text { Fuertes, \& } \\
\text { Gutierrez (2007) }\end{array}$ & $\mathbb{F I}$ & $\begin{array}{l}\text { Instituciones } \\
\text { financieras }\end{array}$ & España & 70 & 2006 & $\begin{array}{c}\text { Desarrolo, financiera, } \\
\text { otra información } \\
\text { financiera }\end{array}$ & 26 & $\begin{array}{l}\mathrm{T}(+) ; \mathrm{R}(+) ; \\
\mathrm{V}(++) ; \mathrm{TE}(+)\end{array}$ & 67 \\
\hline 9 & $\begin{array}{l}\text { Andrikopoulos \& } \\
\text { Diakidis (2007) }\end{array}$ & $|F|$ & Cotizadas & Cyrpus & 140 & 2007 & Items & 39 & $\mathrm{~T}(t) ; \mathrm{R} ; \mathrm{E} ; \mathrm{VM}$ & 68 \\
\hline 10 & $\begin{array}{l}\text { Carcaba \& } \\
\text { Garcia (2008) }\end{array}$ & $\mathbb{F I}$ & Municipios & España & 334 & 2005 & $\begin{array}{l}\text { Conlenido, formato, } \\
\text { interacioion }\end{array}$ & 28 & $\begin{array}{c}T(+), E ; N(+(+) ; \\
C E(+) ; N(-)\end{array}$ & 102 \\
\hline 11 & $\begin{array}{l}\text { Kelton \& Yang } \\
(2008)\end{array}$ & $|F|$ & Cotizadas & Estadds Unidos & 284 & 2003 & Contenido. formato & 36 & $\begin{array}{c}\mathrm{PD}(-) ; \mathrm{DI}(+) ; \\
\mathrm{EF}(+)\end{array}$ & 544 \\
\hline 12 & $\begin{array}{l}\text { Caba, } \\
\text { Rodriguez, \& } \\
\text { López (2008) }\end{array}$ & $\mathrm{IFI}$ & Municipios & España & 65 & 2007 & $\begin{array}{c}\text { Connenido, formato } \\
\text { navegabalidad, diseño } \\
\text { y acoesso }\end{array}$ & 31 & $\begin{array}{c}\text { CP; FE; NEV: P; } \\
\text { PF; CE }(+) ; \\
\text { Al }(+)\end{array}$ & 251 \\
\hline 13 & Gandia (2008) & $|F|$ & Municipios & España & 130 & 2006 & $\begin{array}{c}\text { Presupuestal, } \\
\text { frinanciera, } \\
\text { presentacion y } \\
\text { relación web }\end{array}$ & 88 & $\begin{array}{l}\operatorname{CP}(+), V L_{;} ; ; \\
M P(+) ; i \operatorname{TyE}(+)\end{array}$ & 267 \\
\hline 14 & $\begin{array}{l}\text { Al Arussi, } \\
\text { Selamat, \& } \\
\text { Mohd (2009) } \\
\end{array}$ & ICI & Cotizadas & Malasia & 201 & 2005 & $\begin{array}{l}\text { Ambiental, } \\
\text { financiera }\end{array}$ & 60 & $\begin{array}{c}\mathrm{ED}(+) \mathrm{E} ; \mathrm{TYE} \\
(+) ; \mathrm{R} ; \mathrm{T}(+)\end{array}$ & 127 \\
\hline 15 & Desoky (2009) & $\mathbb{F I}$ & Cotizadas & Egipto & 88 & 2007 & $\begin{array}{l}\text { Contenido, } \\
\text { formato }\end{array}$ & 39 & 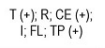 & 61 \\
\hline 16 & $\begin{array}{l}\text { Aly, Simon, \& } \\
\text { Hussainey } \\
\text { (2010) } \\
\end{array}$ & ICI & Cotizadas & Egipto & 62 & 2005 & $\begin{array}{l}\text { Contenido, } \\
\text { presenniación }\end{array}$ & 90 & $\begin{array}{l}\mathrm{R}(+) ; \mathrm{CE}(+) ; \mathrm{I} \\
(+) ; \mathrm{F}_{i} ; \mathrm{I} ; \mathrm{TA}\end{array}$ & 309 \\
\hline \multicolumn{11}{|c|}{ 1/Cantidad de observaciones; $2 /$ Citaciones en Google Scholar, año 2021.} \\
\hline$\#$ & Autores & Tipo & $\begin{array}{c}\text { Tipo de } \\
\text { empresas }\end{array}$ & $\begin{array}{l}\text { Paises o } \\
\text { regiones }\end{array}$ & obs" & $\begin{array}{l}\text { Aña de } \\
\text { estudio }\end{array}$ & Dimensiones & items & $\begin{array}{c}\text { Variables } \\
\text { independien. } \\
\text { tes }^{21}\end{array}$ & $\mathrm{Cit}^{3 s}$ \\
\hline 17 & $\begin{array}{l}\text { Pina et al. } \\
(2010)\end{array}$ & $|F|$ & $\begin{array}{c}\text { Gobiernos } \\
\text { locales }\end{array}$ & Unión Europea & 75 & 2006 & $\begin{array}{l}\text { Conteniob, } \\
\text { presentación }\end{array}$ & 86 & $\begin{array}{l}\mathrm{TC}(+) ; \mathrm{VL} ; \mathrm{NM} ; \\
\mathrm{TA}(+) ; \mathrm{i} A \mathrm{PP}(+) ; \\
\mathrm{Al} ; \mathrm{NC}\end{array}$ & 168 \\
\hline 18 & $\begin{array}{l}\text { Cárcaba \& } \\
\text { Garcla (2010) }\end{array}$ & $|F|$ & Municipios & Esparáa & 334 & 2008 & $\begin{array}{l}\text { Contenico, } \\
\text { formalo: } \\
\text { interaccion }\end{array}$ & 28 & 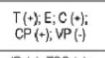 & 136 \\
\hline 19 & $\begin{array}{l}\text { Guillamón, } \\
\text { Bastida, \& } \\
\text { Benito (2011) } \\
\end{array}$ & $|F|$ & Municipios & España & 100 & 2008 & $\begin{array}{c}\text { Financiero, ingresosos y } \\
\text { gastos, deuda }\end{array}$ & 18 & $\begin{array}{l}\mathbb{R} R(+): T R P(+) \\
\text { IDP (t) } P \text {; : MC: } \\
\text { MG }\end{array}$ & 231 \\
\hline 20 & $\begin{array}{l}\text { Haro de } \\
\text { Rosario, Caba, } \\
\text { \& Sánchez } \\
\text { (2012) }\end{array}$ & ICI & $\begin{array}{l}\text { Entidades de } \\
\text { capital de } \\
\text { riesgo }\end{array}$ & España & 89 & 2010 & $\begin{array}{l}\text { Gobiemo orporativo, } \\
\text { activdad economical }\end{array}$ & 45 & 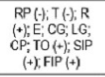 & 4 \\
\hline 21 & $\begin{array}{l}\text { Alali \& Romero } \\
\text { (2012) }\end{array}$ & $|c|$ & Cotizadas & $\begin{array}{c}\text { Argentina: } \\
\text { empresas sue } \\
\text { cotizan enla } \\
\text { Bolsadde Buvonos } \\
\text { Aires }\end{array}$ & 84 & 2009 & 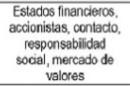 & 53 & $\begin{array}{c}T(+f) R_{i} E_{i} \\
C P(+)\end{array}$ & 82 \\
\hline 22 & $\begin{array}{l}\text { Nurunnabi \& } \\
\text { Hossain (2012) }\end{array}$ & IFI & Cotizadas & Bangladesh & 285 & 2010 & 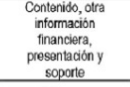 & 56 & $\begin{array}{c}A ; R_{i} ; T_{i} \\
\operatorname{CPF}(t) ; T^{\prime}(t) ; \\
C P ; C A\end{array}$ & 53 \\
\hline 23 & $\begin{array}{l}\text { Maldonado, } \\
\text { Escobar, \& } \\
\text { Marin (2012) }\end{array}$ & $|C|$ & Cotizadas & México y Chile & 75 & 2010 & $\begin{array}{c}\text { Intormes } \\
\text { Genenales, } \\
\text { Inversionistas }\end{array}$ & 18 & $T(-) ; R ; L$ & 12 \\
\hline 24 & $\begin{array}{l}\text { Andrikopoulos et } \\
\text { al. (2013) }\end{array}$ & $|F|$ & Cotizadas & $\begin{array}{l}\text { NYSE, Nastaq, } \\
\text { Bolsas de } \\
\text { Londres, Bolsa } \\
\text { ce Singapar, } \\
\text { Bolsa de OSio }\end{array}$ & 171 & 2010 & $\begin{array}{l}\text { Contenico } \\
\text { Presentááón }\end{array}$ & 31 & $\underset{E(-) ; C P}{T(-):}$ & 47 \\
\hline 25 & Yusuf (2013) & $|F|$ & Cotizadas & Nigeria & 84 & 2013 & $\begin{array}{c}\text { Contenido, } \\
\text { usabilidad, apoyo y } \\
\text { oportunidad }\end{array}$ & 18 & 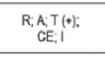 & 9 \\
\hline 26 & $\begin{array}{l}\text { Gömez \& } \\
\text { Montesinos } \\
(2014)\end{array}$ & $\mathbf{I F I}$ & $\begin{array}{c}\text { Departamento } \\
\text { s }\end{array}$ & Colombia & 33 & 2012 & $\begin{array}{c}\text { Contenido } \\
\text { caractenisticas, } \\
\text { navegabilidad y } \\
\text { acceso }\end{array}$ & 33 & 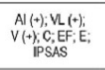 & 18 \\
\hline 27 & $\begin{array}{l}\text { Fuertes, Cuellar, } \\
\text { \& Pelayo (2014) }\end{array}$ & ICI & Cotizadas & $\begin{array}{l}\text { Argentina, } \\
\text { Mexios, chile }\end{array}$ & 76 & 2008 & 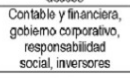 & 31 & 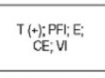 & 24 \\
\hline 28 & Aqel (2014) & $\mathbb{F} \mid$ & Cotizadas & Estambel & 263 & 2012 & Index & 44 & $T(+) ; E ; R(+) ; L$ & 36 \\
\hline 29 & $\begin{array}{l}\text { Dolinšek, } \\
\text { Tomininc, \& } \\
\text { Skerbinjek } \\
\text { (2014) }\end{array}$ & $\mathbb{F I}$ & $\begin{array}{l}\text { Grandes } \\
\text { empresas }\end{array}$ & Estovenia & 110 & 2012 & $\begin{array}{c}\text { Contenido } \\
\text { presentlación }\end{array}$ & 50 & $\begin{array}{l}T(t): R ; F L(+) ; \\
C P(+) ; A ;(+)\end{array}$ & 53 \\
\hline 30 & Bowrin (2015) & ICI & Cotizadas & $\begin{array}{l}\text { Paises del } \\
\text { Canibe }\end{array}$ & 65 & 2009 & $\begin{array}{l}\text { Crediblidad, } \\
\text { responsabilidad } \\
\text { social, } \\
\text { puntualidad } \\
\text { usabildad, } \\
\text { caracteristicas }\end{array}$ & 107 & 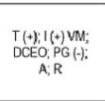 & 19 \\
\hline 31 & $\begin{array}{l}\text { Pinto \& Ng } \\
\text { Picoto (2016) }\end{array}$ & $|F|$ & Cotizadas & $\begin{array}{c}\text { FTSE100 Raino } \\
\text { Unido }\end{array}$ & 78 & 2014 & $\begin{array}{c}\text { Conteniciob } \\
\text { presentación }\end{array}$ & 63 & $A(t) ; R S(t)$ & 19 \\
\hline 32 & $\begin{array}{l}\text { Omran \& } \\
\text { Ramalhony } \\
\text { (2016) }\end{array}$ & $\mathbb{F} \mid$ & Cotizadas & Mauricio (Ática) & 34 & $\begin{array}{l}\text { No } \\
\text { indica }\end{array}$ & 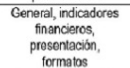 & 52 & 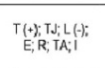 & 18 \\
\hline 33 & $\begin{array}{l}\text { Ahmed, Burton, } \\
\text { \& Dunne (2017) }\end{array}$ & $|C|$ & № cobzadas & Egipto & 172 & $\begin{array}{l}2010- \\
2011\end{array}$ & $\begin{array}{c}\text { Contenidis, spopoter, } \\
\text { prosentiaciono }\end{array}$ & 110 & 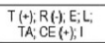 & 30 \\
\hline
\end{tabular}




\begin{tabular}{|c|c|c|c|c|c|c|c|c|c|c|}
\hline \# & Autores & Tipo & $\begin{array}{c}\text { Tipo de } \\
\text { empresas }\end{array}$ & $\begin{array}{l}\text { Paises } 0 \\
\text { regiones }\end{array}$ & Obs" & $\begin{array}{l}\text { Año de } \\
\text { estudio }\end{array}$ & Dimensiones & Ittems & $\begin{array}{c}\text { Variables } \\
\text { independien- } \\
\text { tes }^{2}\end{array}$ & $\mathrm{Cit}^{y t}$ \\
\hline 34 & Yassin (2017) & $|F|$ & Cotizadas & Jordania & 228 & 2011 & $\begin{array}{l}\text { Contenido, } \\
\text { Formato }\end{array}$ & 30 & 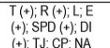 & 15 \\
\hline 35 & $\begin{array}{l}\text { Aboutera \& } \\
\text { Hussein (2017) }\end{array}$ & IFI & Cotizadas & Egipto & 133 & $\begin{array}{r}2014 y \\
2016\end{array}$ & $\begin{array}{c}\text { Contenido, ota } \\
\text { información } \\
\text { financiera, soporte }\end{array}$ & 56 & $\begin{array}{c}T(+) ; R ; L: E ; A ; \\
C P ; T A(+)\end{array}$ & 2 \\
\hline 36 & $\begin{array}{l}\text { Pivac, Vuko, \& } \\
\text { Cular (2017) }\end{array}$ & $\mathrm{ICl}$ & Cotizadas & 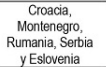 & 150 & 2012 & Contenido & 41 & $E ; R ; C ; L G(+)$ & 28 \\
\hline 37 & $\begin{array}{l}\text { Ariff, Bin- } \\
\text { Ghanem, \& } \\
\text { Hashim (2018) }\end{array}$ & IFI & $\begin{array}{l}\text { Sevicios } \\
\text { financieros }\end{array}$ & $\begin{array}{l}\text { Babrein, Kuwait, } \\
\text { Omá, Quatar, } \\
\text { Arabia Suaditay } \\
\text { Emiratos Árabes } \\
\end{array}$ & 152 & 2012 & $\begin{array}{l}\text { Presenentaacón, } \\
\text { contenido }\end{array}$ & 35 & 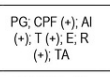 & 11 \\
\hline 38 & $\begin{array}{l}\text { Khriech, Khouri, } \\
\text { \& Ghorbel (2018) }\end{array}$ & ICI & Cotizadas & 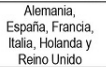 & 197 & $\begin{array}{l}2011- \\
2012\end{array}$ & $\begin{array}{c}\text { Contenico, } \\
\text { oportunidad } \\
\text { tecnologia, soporte }\end{array}$ & 133 & 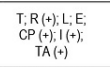 & 0 \\
\hline 39 & $\begin{array}{l}\text { Dolinšek \& Lutar } \\
\text { (2018) }\end{array}$ & IFI & Cotizadas & Eslovenia & 192 & 2015 & IFR-content & 50 & $\begin{array}{l}T(+) ; R ; A ; C P \\
(+; i) i(+)\end{array}$ & 18 \\
\hline 40 & $\begin{array}{l}\text { Noreno, Gras, \& } \\
\text { Henandez (2019) }\end{array}$ & $|F|$ & Municipios & España & 1806 & 2016 & $\begin{array}{c}\text { Contenico, } \\
\text { prossentación, } \\
\text { Interacción }\end{array}$ & 15 & 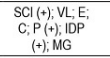 & 2 \\
\hline 41 & $\begin{array}{l}\text { Keliwon et al. } \\
\text { (2018) }\end{array}$ & IFI & Cotizadas & Malasia & 320 & 2012 & 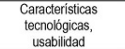 & 17 & $T(+) ; R ; E ; C G$ & 10 \\
\hline 42 & Sia et al. (2018) & ICI & Cotizadas & Malasia & 582 & 2013 & $\begin{array}{l}\begin{array}{l}\text { Formato, } \\
\text { contenido }\end{array} \\
\end{array}$ & 39 & $\mathrm{~T} ; \mathrm{A}(-) ; \mathrm{CG}(+)$ & 6 \\
\hline 43 & $\begin{array}{l}\text { Bananuka } \\
(2019)\end{array}$ & IFI & $\begin{array}{l}\text { Sevvicios } \\
\text { financieros }\end{array}$ & Ugarda & 40 & No indica & $\begin{array}{l}\text { Fuerzas isomorfas, } \\
\text { capital intelectual, } \\
\text { financieros }\end{array}$ & 57 & $\begin{array}{l}\text { AJ;; FRCA (t); } \\
\text { ISC (I); IN); } \\
\text { ISM }\end{array}$ & 6 \\
\hline 44 & $\begin{array}{l}\text { Sandhu \& Singh } \\
\text { (2019) }\end{array}$ & $\mathrm{ICl}$ & Cotizadas & India & 140 & 2015 & 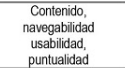 & 136 & 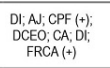 & 5 \\
\hline 45 & $\begin{array}{l}\text { A-Sarrawi \& } \\
\text { Reyad (2019) }\end{array}$ & |FI & $\begin{array}{l}\text { Bancos } \\
\text { islámicos }\end{array}$ & Paises del Gollio & 48 & 2016 & $\begin{array}{c}\text { Contenido, } \\
\text { presentación }\end{array}$ & 90 & $\begin{array}{l}\mathrm{ROE}(+) ; \mathrm{ROA} \\
(+) ; \mathrm{GA} ; \mathrm{T} ; \mathrm{E}\end{array}$ & 5 \\
\hline 46 & $\begin{array}{l}\text { Xang \& Birt } \\
\text { (2021) }\end{array}$ & $\mathrm{ICl}$ & Cotizadas & Australia & 200 & 2018 & $\begin{array}{l}\text { Inversor, RS, } \\
\text { gobiemono corortivo, } \\
\text { puntulidad, } \\
\text { sevicios, , edes } \\
\text { sociales, usabilitlad }\end{array}$ & 60 & 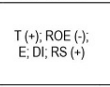 & 2 \\
\hline \multicolumn{11}{|c|}{ 1/ Cantidad de observaciones. ${ }^{2} /$ Ver siglas en la parte final de la tabla. ${ }^{3} /$ Citaciones en Google Scholar, año 2021.} \\
\hline & 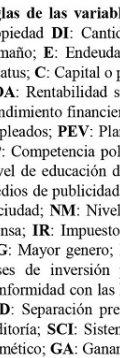 & $\begin{array}{l}\text { amiento } \\
\text { patrimo } \\
\text { sobre A } \\
\text { ero; NA } \\
\text { anes de } \\
\text { litica; } \\
\text { de los v } \\
\text { t; TyE: } \\
\text { i de ma } \\
\text { os recau } \\
\text { RP: Re } \\
\text { prefere } \\
\text { IPSAS } \\
\text { esidente } \\
\text { ma de } \\
\text { incia po }\end{array}$ & 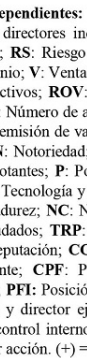 & 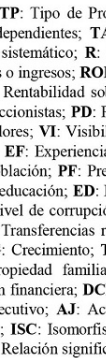 & 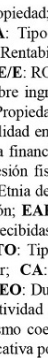 & 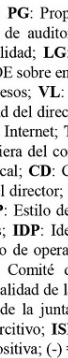 & 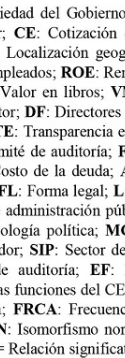 & $\begin{array}{l}\text { ráfica, } \\
\text { ntabilid } \\
\text { M: Val } \\
\text { de fam } \\
\text { lectrón } \\
\text { FE: Fon } \\
\text { AI: Aco } \\
\therefore \text { Liqui } \\
\text { blica; } \\
\text { C: Muj }\end{array}$ & 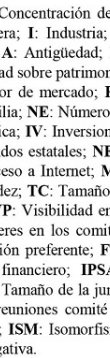 & $\begin{array}{l}\text { le la } \\
\text {; T: } \\
\text { Et: } \\
\text { otio; } \\
\text { RF: } \\
\text { o de } \\
\text { nes; } \\
\text { EV: } \\
\text { MP: } \\
\text { o de } \\
\text { en la } \\
\text { ités; } \\
\text { FiP: } \\
\text { ASS: } \\
\text { unta; } \\
\text { éde } \\
\text { smo }\end{array}$ \\
\hline
\end{tabular}

Fuente: elaboración propia.

En cuanto a la publicación de trabajos relacionados con índices relativos a IFI, en la tabla 2 se presenta el listado de las revistas, su nivel de impacto y cantidad de publicaciones en relación con los artículos de estudio. La revista con mayor número de artículos es Online Information Review, con cinco trabajos, seguida por Journal of Accounting and Public Policy, Local Government Studies, Research Journal of Finance and Accounting. Journal of accounting in emerging economies, cada una con dos artículos. Cabe decir, que las revistas que suelen publicar sobre este tema, generalmente están ranqueadas entre aquellas con calificaciones de mayor impacto. 
TABLA 2

Listado de las revistas

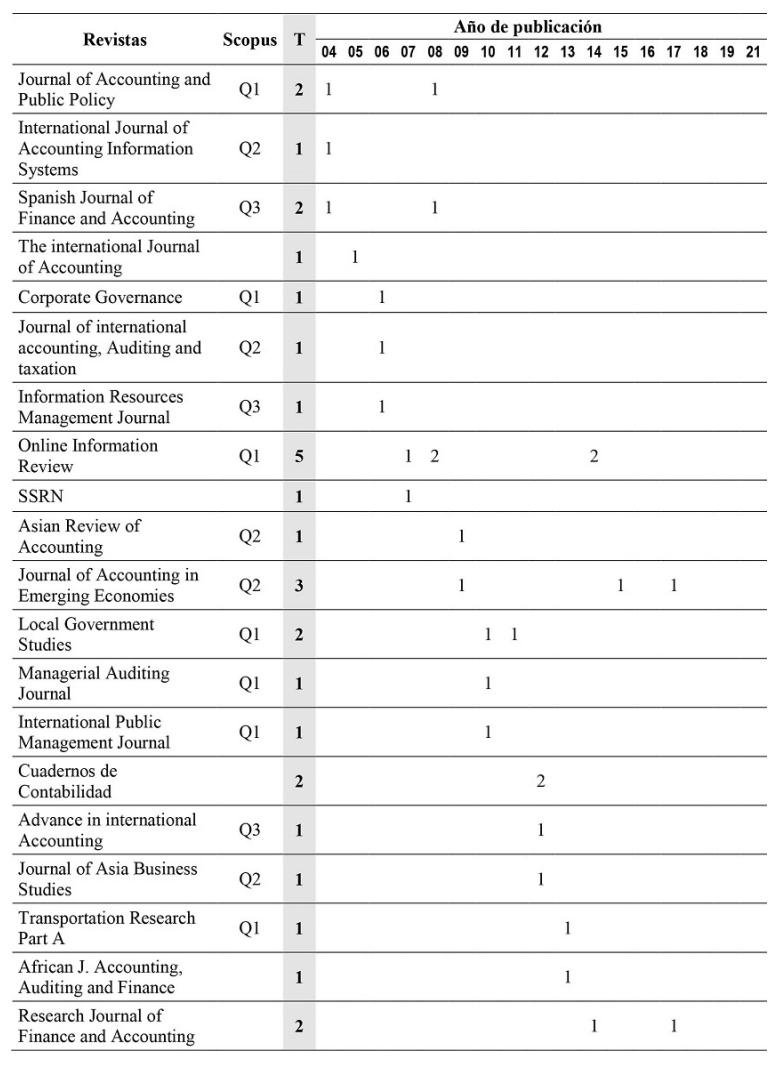

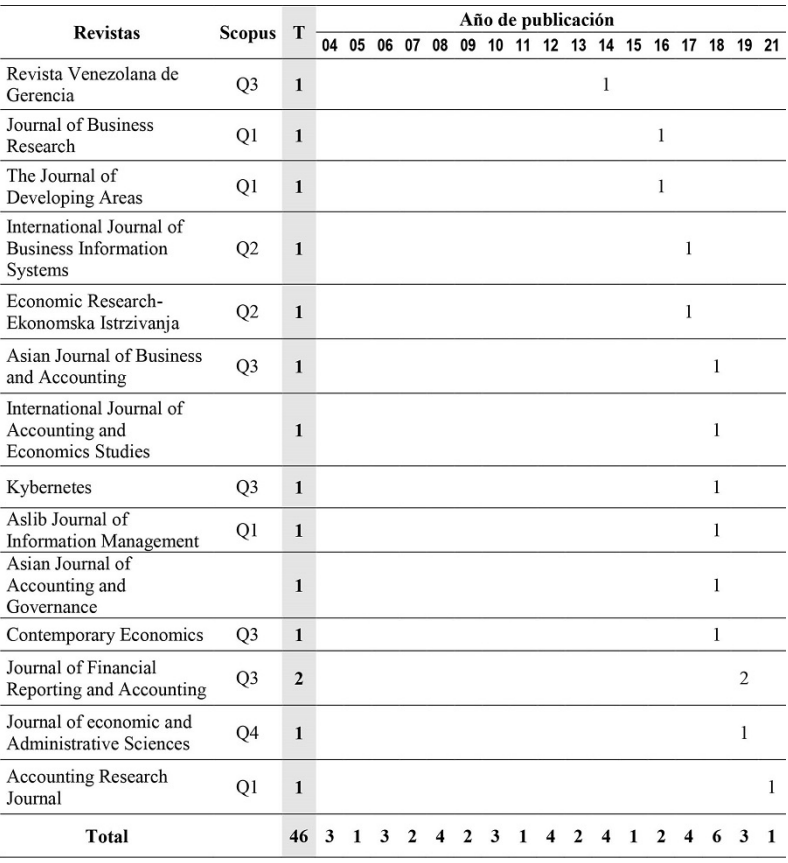

Fuente: elaboración propia.

\section{Características comunes de los índices}

Los índices son una proxy a partir de la cual es posible establecer los niveles de divulgación y transparencia de la información financiera, dado que estos incluyen conceptos abstractos que no pueden ser observados de manera directa (Ali Khan \& Ismael, 2012b; Maldonado-García et al., 2012; Mokhtar, 2017; Serrano-Cinca, Fuertes-Callén, \& Gutierrez-Nieto, 2007). "El índice tiene como objetivo mostrar el nivel de divulgación 
en un conjunto de cuentas de la empresa” (Ali Khan \& Ismael, 2011, p. 10). El índice está formado por ítems, siendo una variable dicotómica, que toma un valor de uno (1) en caso de existencia de la variable observada y cero (0) en caso de inexistencia. En algunos trabajos se acepta una calificación de 0,5 cuando se cumple parcialmente el criterio del ítem (Pina et al., 2010). Estos índices pueden ser ponderados o no ponderados, el primero da un porcentaje diferente a las dimensiones e ítems según la importancia que requiere la investigación, el no ponderado da el mismo valor a cada ítem. Este último es el más frecuente en las investigaciones revisadas.

\section{Dimensiones utilizadas en los índices}

Las dimensiones son las variables dependientes que agrupan los ítems con los cuales se miden los niveles de divulgación. Un índice puede tener varias dimensiones que determinan un constructo de los contenidos que se van a avaluar en el estudio. Las dimensiones más comunes en los índices de IFI o Informes Corporativos en Internet son contenido y presentación (ver tabla 1). La primera está dirigida a calificar la existencia de información financiera y no financiera importante para las decisiones de los stakeholders; la segunda generalmente evalúa el diseño, amigabilidad y la facilidad de la página web para encontrar y obtener los contenidos (Ali Khan \& Ismael, 2011). Algunos de los índices estudiados mostraron subdimensiones en contenido y presentación (Sandhu \& Singh, 2019).

No obstante, las dimensiones dependen del objetivo de la investigación, pues algunos trabajos buscan observar características de la información como homogeneidad, puntualidad, responsabilidad y, para ello, plantean otras dimensiones que permiten separar las variables dependientes relacionadas (Sandhu \& Singh, 2019; Xiang \& Birt, 2021). Dichos trabajos se proponen observar otros elementos como gobierno corporativo, otra información financiera, medio ambiente, tecnología, interacción con usuarios, accionistas, empleados, mercado de valores, entre otros (Alali \& Romero, 2012). El número promedio de las dimensiones abordadas en los índices hallados se establece en tres dimensiones. Generalmente, cada dimensión del índice es explicada por separado con los determinantes elegidos en la investigación, lo cual, puede entregar resultados distintos al resultado total del índice. En algunos trabajos se presenta además, la correlación de los resultados de las dimensiones entre ellas (Desoky, 2009; Gómez-Villegas \& Montesinos Julve, 2014).

\section{Ítems utilizados en los índices}

Los ítems son los elementos verificables, observables directamente y constatables, que están relacionados con el constructo de la dimensión a evaluar. Estos, a su vez, se asocian a cada dimensión y se establecen según el contexto de investigación. De los artículos revisados, el índice con más ítems tiene 160 y el de menos 18 (ver tabla 1). El promedio de ítems utilizados en los artículos revisados es de 55 ítems. Esto es importante porque la profundidad de las prácticas a observar determinará la calidad de la divulgación de información realizada. La literatura no ha reconocido que la complejidad de la comunicación en Internet no implica que más información conlleve mayor transparencia (Lev, 2018).

Los ítems de contenido financiero más comunes son las preguntas sobre: presencia en las páginas corporativas de los distintos estados financieros, el informe de auditoría, las políticas contables, las notas, la presentación de informes financieros de años anteriores, la presentación de informes intermedios, indicadores financieros, informes por segmentos, los memoriales, la estructura de propiedad, dividendos y los informes de gestión de riesgo. En la dimensión de presentación se pregunta sobre: presencia de los informes y la página web en distintos idiomas y formatos, link de acceso a los informes financieros, presencia de gráficos para explicación de los informes, sitios de ayuda, mapas del sitio, buscador interno, preguntas frecuentes, noticias, 
enlaces externos, dirección, correos, número de teléfonos y contáctenos. Los ítems varían dependiendo del objetivo de estudio de la investigación.

\section{Teorías utilizadas en los estudios de IFI}

Como se muestra en la figura 1, la teoría regularmente utilizada para explicar las relaciones significativas de los resultados de IFI es la teoría de agencia, de los 46 artículos analizados, tan solo tres no recurren a ella. La teoría de agencia es clave para la explicación de los IFI, pues el conflicto de interés, el riesgo moral que originan los costos de agencia, dada la asimetría de información, ha demostrado que incentiva la divulgación en las páginas web corporativas (Mokhtar, 2017). Por su parte, la teoría de la señalización con un total de 22 trabajos es la segunda teoría más empleada en la literatura analizada, lo cual podría explicarse, pues plantea que las empresas con mejores resultados o reputación preferirán divulgar más información para ofrecer confianza ante los accionistas (Aly, Simon, \& Hussainey, 2010; Andrikopoulos, Merika, Triantafyllou et al., 2013).

La asimetría de información se encuentra en ocho artículos. Esta teoría enmarca las diferencias en los niveles de acceso a la información entre la partes de un acuerdo (Rivera-Arrubla, Zorio-Grima, \& GarcíaBenau, 2017), y generalmente, se encuentra referenciada junto a la teoría de agencia. Por su parte, la teoría de legitimidad se encuentra en siete artículos, la cual establece que las empresas podrían mejorar la calidad de la divulgación para tener una mayor aceptación social (Maldonado-García et al., 2012). La teoría de la innovación y la difusión con seis trabajos ocupa el quinto lugar, explica la tecnología como un agente de cambio que influencia su uso en determinados contextos empresariales (Xiao et al., 2004). Asimismo, la teoría de los costos políticos se encuentra en seis referencias. Esta teoría se enfoca en la presión social como un indicador de divulgación (Fuertes-Callén et al., 2014). Finalmente, y con una menor frecuencia se encuentran otras teorías como la institucional, la stakeholder, de la economía política, de relevancia cultural, de los incentivos, del estado de derecho, la teoría positiva de la contabilidad, de la elección pública y la teoría de gobierno corporativo.

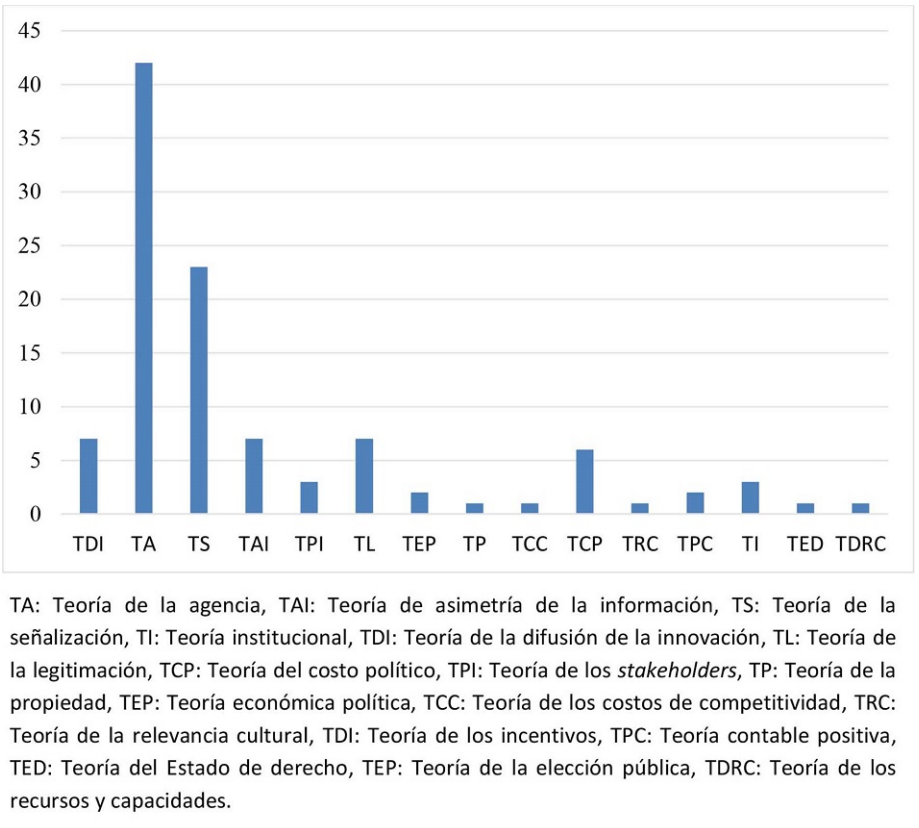

FIGURA 1

Teorías utilizadas en los artículos seleccionados Fuente: elaboración propia. 


\section{Metodologías utilizadas en los estudios de IFI}

Los modelos explicativos de los determinantes IFI e Informes Corporativos en Internet proporcionan evidencia empírica sobre la relación significativa entre las variables independientes y el índice. A esto se debe la utilización de metodologías de estadística inferencial para relacionar los resultados de las variables encontradas. La mayoría de los trabajos estudiados utilizan modelos de regresión múltiple o modelos de regresión lineal por Mínimos Cuadrados Ordinarios (MCO). Por su parte, unos pocos trabajos recurren a metodologías poco utilizadas, de las cuales, cada una fue hallada en solo un trabajo: método de momentos generalizados (Andrikopoulos et al., 2013), pruebas no paramétricas de comparación de medianas (Maldonado-García et al., 2012) regresión logística binaria (Dolinšek \& Lutar-Skerbinjek, 2018), modelo de ecuación estructural (Serrano-Cinca, Fuertes-Callén, \& Gutiérrez-Nieto, 2007), método PROMETHEE (Pivac et al., 2017) (ver figura 2). Aunque la investigación cuantitativa tiene limitaciones por la lógica interna de las regresiones, sus resultados permiten conocer tendencias y relaciones que ayudan a explicar dinámicas que se asumen reiterativas o estructurales.

Para la recolección de la información específica algunos trabajos utilizaron la metodología del análisis de contenido, sin embargo, en la mayoría de trabajos no se establece una metodología concreta para este proceso.

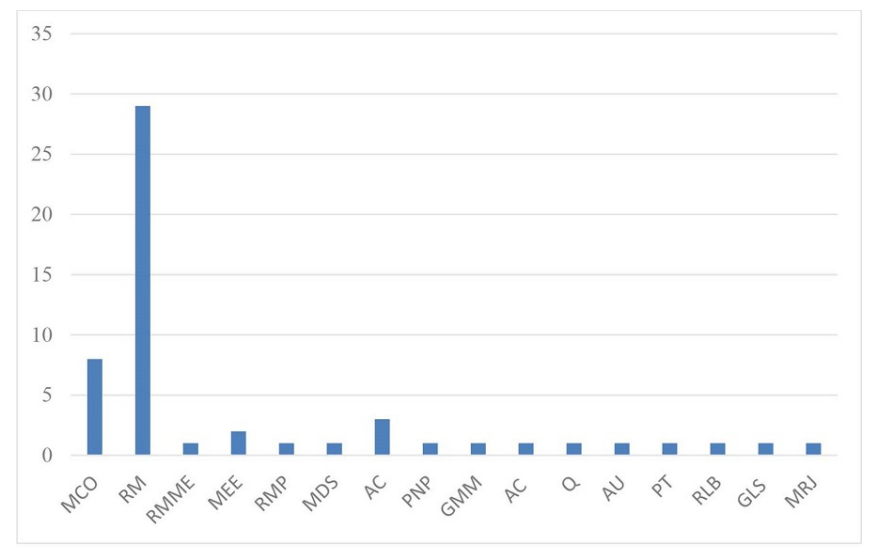

MCO: Regresión por mínimos cuadrados ordinarios, RM: Regresión multivariante, RMME: Regresión múltiple método por etapas, MEE: Modelo de ecuación estructural, RMP: Regresión múltiple ponderada, MDS: Análisis multivariante escalamiento multidimensional, AC: Análisis de contenido, PNP: Prueba no paramétrica de comparación de medianas, GMM: Método de momentos generalizados, Q: Q de Tobin, AU: Análisis univariado, PT: Método PROMETHEE de criterios múltiples, RLB: Regresión logística binaria, GLS: Regresión por mínimos cuadrados generalizados, MRJ: Modelo de regresión jerárquica.

\section{FIGURA 2}

Metodologías utilizadas

Fuente: elaboración propia.

\section{Determinantes de la divulgación de IFI}

Los determinantes hacen referencia a variables independientes que logran explicar la variable dependiente, explican el nivel de significancia o el efecto que dicha variable puede tener sobre la variable dependiente. Por lo tanto, la correlación entre estas variables es importante, en tanto incluye o excluye una variable como explicativa en términos de impactar la elección de divulgar mayor información en los reportes financieros, lo que permite interpretar los posibles comportamientos del mercado con respecto al problema de estudio.

Para la explicación de la divulgación de los IFI los determinantes utilizados son muy variados. En general, son variables relacionadas con características de la empresa como tamaño, rentabilidad, crecimiento, endeudamiento, liquidez, tipo de auditor, antigüedad, concentración de la propiedad, ganancia por acción, porcentaje de propiedad del gobierno. Igualmente, se encuentran variables de gobierno corporativo como directores independientes, dualidad del CEO, cantidad de miembros de los comité y tamaño de la junta, 
y otras variables institucionales como región, país, industria, sector, penetración de Internet en el país y corrupción del país (Debreceny \& Gray, 1999). En los trabajos sobre entidades estatales se encuentran usualmente variables como población, ideología política, riqueza del municipio, género, sistema de control interno, entre otras (ver tabla 1).

En cuanto a las variables independientes de los artículos seleccionados, si bien existen algunas comunes en varios estudios, otras han sido definidas para el contexto y problema específico de investigación (ver figura 3). Este hecho hace difícil equiparar y comparar los estudios entre sí y es un vacío de investigación relevante. Asimismo, se requiere definir y testear criterios de discriminación del contexto (cultura, estructura institucional, nivel de desarrollo, entre otros), para profundizar la explicación de la divulgación IFI.

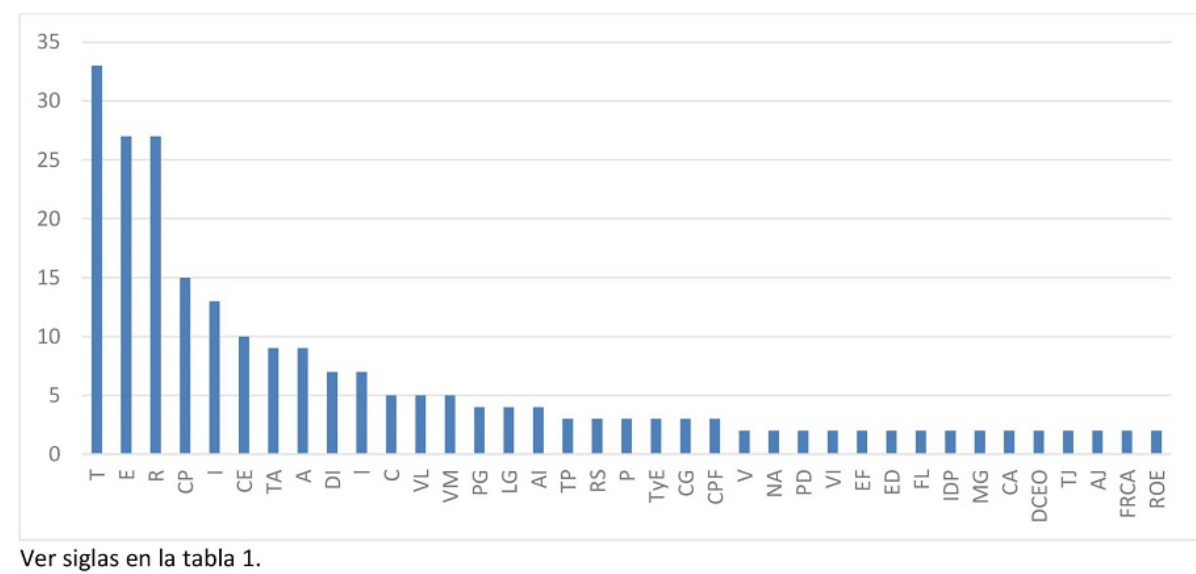

FIGURA 3

Variables independientes más utilizadas

Fuente: elaboración propia.

\section{Discusión}

La figura 4 fue generada usando el software VOSviewer, y a partir de la información contenida en los títulos, resúmenes y palabras clave obtenidos de las bases de datos de Scopus. Web of Science (Arango, Ceballos, \& Osorio, 2020). El mapa de conceptos muestra que la investigación sobre información financiera en Internet en la última década ha estado relacionada con los temas de gobierno corporativo, e-goverment, así, como la divulgación de información voluntaria. Estos resultados manifiestan la tendencia a observar de manera integral el tema de divulgación de información en Internet. Esto es importante, dado que podría ampliar la visión de transparencia hacia otros stakeholders distintos a los inversionistas y busca articular la lógica del pensamiento y el reporte integrado en la divulgación de información por medios electrónicos (Lodhia \& Stone, 2017). 


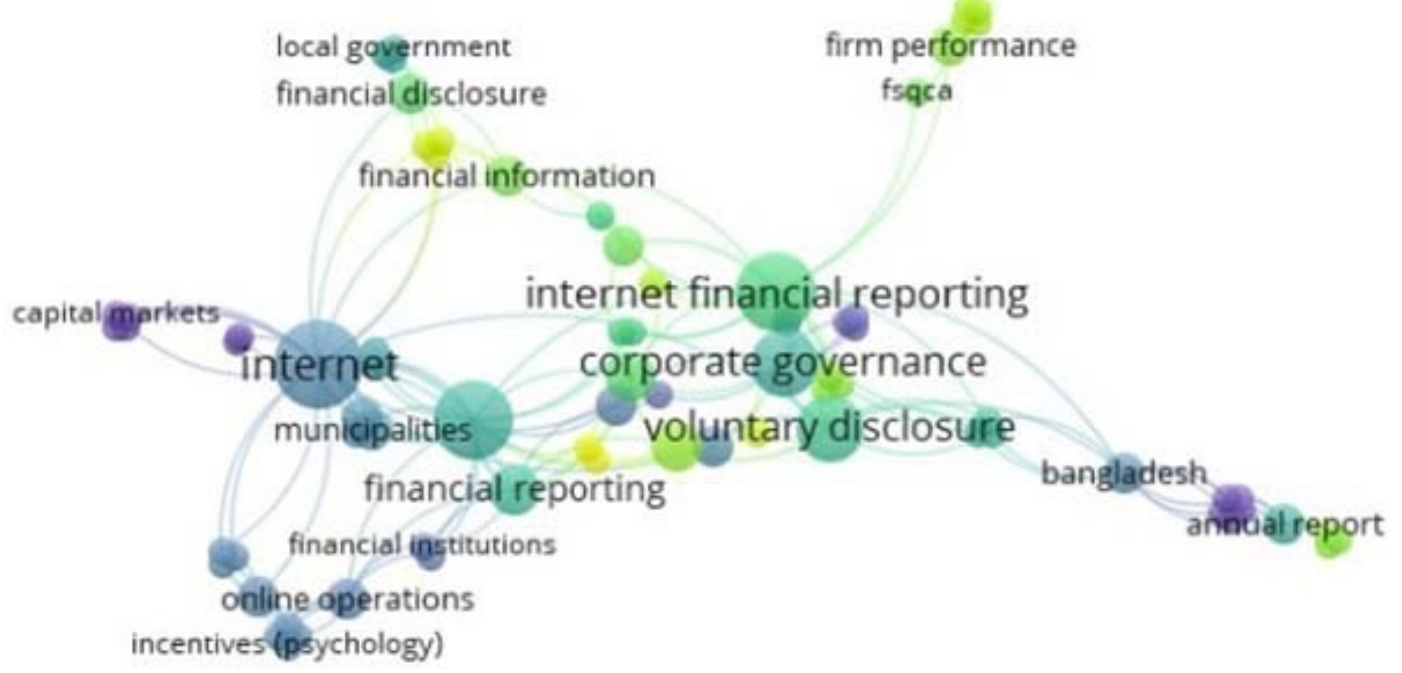

FIGURA 4

Mapa de conceptos

Fuente: elaboración propia.

La figura 5 permite visualizar los trabajos desarrollados en la primera década de estudio que alimentan el soporte académico en la investigación sobre informes financieros y no financieros en Internet. Trabajos como los de Xiao (2004), Marston (2004), Kelton \& Yang (2008) constituyen los estudios de mayor referencia en cuanto a las empresas privadas. Por su parte, los trabajos de Caba, Rodríguez \& López (2008) y Gandía (2008), influyen de forma importante en los análisis en materia estatal. La diferenciación de las prácticas de divulgación de información entre el sector privado y el gobierno es determinante para reconocer que el gobierno electrónico no solo busca eficiencia y rentabilidad, sino también inclusión social y equidad (Twizeyimana \& Andersson, 2019). En términos generales, los diseños de los índices de estos trabajos se caracterizan por ser inteligibles y claros, cuestión que contribuye a explicar porqué se han convertido en referencias obligadas en la investigación académica sobre índices de IFI.

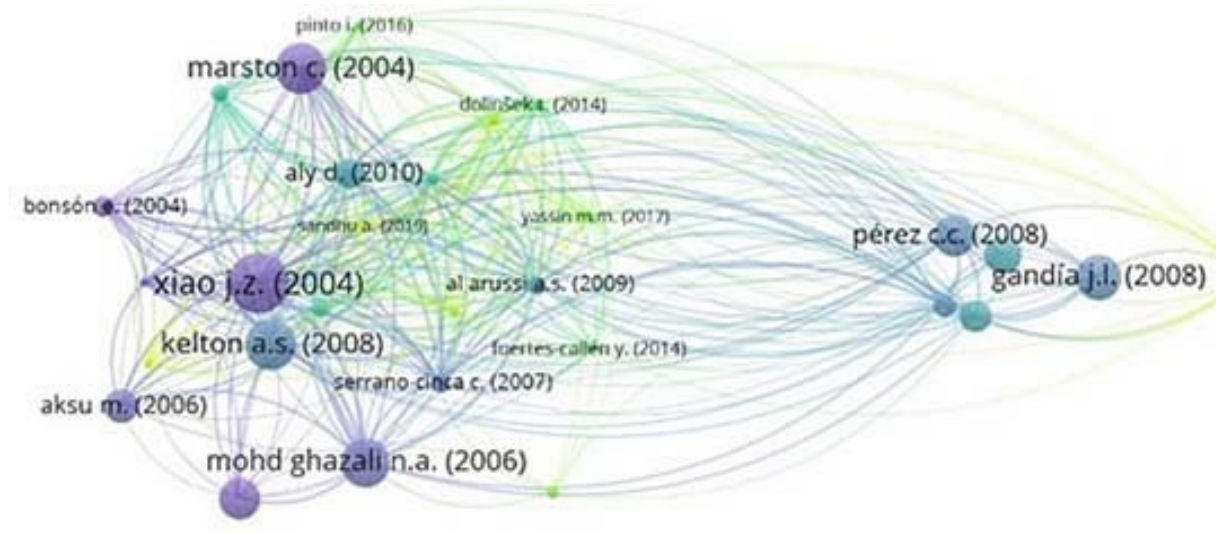

FIGURA 5

Mapa de redes de autores

Fuente: elaboración propia.

Para Ali Khan \& Ismael (2010, 2012b), la construcción de un índice puede implicar juicios subjetivos de los investigadores, además, plantean que los índices deberían ser más completos y homogéneos, dado que la variabilidad de las dimensiones e ítems, no permite la comparabilidad de los resultados. No obstante, otros autores plantean que el diseño de la investigación debe corresponder a los propósitos del trabajo, los cuales son diversos, por lo que cada índice sería distinto. Algunos autores señalan que la poca homogeneidad en la construcción y características de los índices encontrados en este estudio obedece a una falta de coordinación 
entre las investigaciones en IFI. Mientras que para otros esta diversidad sería producto de la evolución en la que se encuentra este campo de investigación (Mokhtar, 2017).

Pretender la unificación del diseño de índices para los estudios de investigación en IFI, en el punto de evolución en el que estos se encuentran, podría no ser adecuado. Esto, debido a que probablemente quedarían por fuera componentes importantes que toman fuerza en las estrategias de divulgación de información, así como aspectos contextuales clave para la asimilación de las TIC y la innovación social. Por lo tanto, este trabajo plantea continuar la investigación para comprender la evolución de la divulgación IFI, la discusión sobre la elaboración de estándares para la divulgación de información en Internet, así como, sus efectos en la transparencia y la generación de valor en los sectores privado y público (Xiang \& Birt, 2021). Además, se debe tener en cuenta que los estudios sobre divulgación IFI se han realizado generalmente para describir, comparar y explicar los aportes a la construcción de valor para los accionistas, pero pocas veces se han planteado en términos de valor para otros actores de la organización, como son los ciudadanos, los políticos, los trabajadores, los proveedores y acreedores, actores que podría tenerse en cuenta en las investigaciones de IFI (Twizeyimana \& Andersson, 2019).

\section{Conclusiones}

Esta revisión sistemática aporta elementos fundamentales para los investigadores en IFI, en cuanto a la estructura de los diseños de índices. Se expuso una síntesis de los trabajos identificando las características, dimensiones, ítems, teorías, metodologías y los hallazgos más significativos que explican la divulgación de reportes financieros en Internet por parte de las empresas. Asimismo, este trabajo podría apoyar a las empresas en la identificación de aquellos elementos que deberían integrar en sus páginas web corporativas, con el propósito de mejorar, tanto la divulgación de información financiera en Internet, como la percepción de transparencia ante sus stakeholders. Además, este trabajo es relevante para los reguladores, dada la discusión actual sobre la necesidad de estándares que contemplen la divulgación de información en Internet.

Los estudios sobre IFI pueden ser clasificados de manera básica entre aquellos que identifican las características o elementos presentes en los informes divulgados, y aquellos que buscan establecer el nivel o la cantidad de información divulgada y sus posibles factores determinantes. Para algunos autores, más información no significa más transparencia (Lev, 2018). Por ello, el problema de la divulgación en Internet no debería cifrarse en la cantidad de la información divulgada. No obstante, dados los procesos de evolución alrededor de las prácticas y formas de divulgación, las investigaciones a futuro podrían ampliarse hacia la revisión de otros elementos como la calidad, la relevancia y la oportunidad de la información revelada, para conseguir más transparencia y mejor rendición de cuentas. Asimismo, sería interesante evaluar las propuestas de índices IFI que describe este trabajo a partir de investigaciones que apliquen alguno de los índices, sistematizando y contrastando los resultados de autores que recurrieron al mismo índice. Sería importante indagar por la determinación de elección, los contextos socio-culturales en los que fue aplicado y adecuaciones realizadas.

\section{Consideraciones éticas}

Dada la naturaleza del estudio no requiere de aval ético. 


\section{Contribución de los autores}

Este artículo se deriva del estado del arte del trabajo de tesis doctoral de Carlos Augusto Rincón Soto, dirigido por el profesor Mauricio Gómez Villegas y la profesora Yaismir Adriana Rivera Arrubla.

\section{Financiación}

No hubo ningún tipo de financiación.

\section{Conflictos de interés}

Los autores declaran no tener ningún tipo de conflicto de interés.

\section{Referencias}

Aboutera, L., \& Hussein, A. (2017). Determinants of internet financial reporting by Egyptian. Research Journal of Finance and Accounting, 8(10), 28-39. https://www.iiste.org/Journals/index.php/RJFA/article/view/37284

Ahmed, A. H., Burton, B. M., \& Dunne, T. M. (2017). The determinants of corporate internet reporting in Egypt: An exploratory analysis. Journal of Accounting in Emerging Economies, 7(1), 35-60. https://doi.org/10.1108/j aee-04-2015-0024

Akhtaruddin, M. (2005). Corporate mandatory disclosure practices in Bangladesh. International Journal of Accounting, 40(4), 399-422. https://doi.org/10.1016/j.intacc.2005.09.007

Aksu, M., \& Kosedag, A. (2006). Transparency and disclosure scores and their determinants in the Istanbul Stock Exchange. Corporate Governance, 14(4), 277-296. https://doi.org/10.1111/j.1467-8683.2006.00507.x

Al-Sartawi, A. M. A. M., \& Reyad, S. M. R. (2019). The relationship between the extent of online financial disclosure and profitability of Islamic banks. Journal of Financial Reporting and Accounting, 17(2), 343-362. https://doi .org/10.1108/JFRA-11-2017-0103

Al Arussi, A. S., Selamat, M. H., \& Mohd, M. (2009). Determinants of financial and environmental disclosures through the internet by Malaysian companies. Asian Review of Accounting, 17(1), 59-76. https://doi.org/10.1108/132 17340910956513

Alali, F., \& Romero, S. (2012). The use of the Internet for corporate reporting in the Mercosur (Southern common market): The Argentina case. Advances in Accounting, 28(1), 157-167. https://doi.org/10.1016/j.adiac.2012.0 3.009

Ali Khan, M. N., \& Ismael, N. (2010). A Study on the Index of Internet. IPBJ, 2(1), 1-23. https://www.researchgat e.net/publication/259704688

Ali Khan, M. N., \& Ismael, N. (2011). The use of disclosure indices in internet financial reporting research. Journal of Global Business and Economics, 3(1), 157-173. https://www.researchgate.net/publication/227439503

Ali Khan, M. N., \& Ismael, N. (2012a). A Review of E-Financial Reporting Research.Journal of Internet and E-Business Studies, (January), 1-16. https://doi.org/10.5171/2012.798113

Ali Khan, M. N., \& Ismael, N. (2012b). An empirical study on the indexes of internet financial reporting: The case of Malaysia. African Journal of Business Management, 6(5), 2086-2100. https://doi.org/10.5897/AJBM11.1596

Aly, D., Simon, J., \& Hussainey, K. (2010). Determinants of corporate internet reporting: Evidence from Egypt. Managerial Auditing Journal, 25(2), 182-202. https://doi.org/10.1108/02686901011008972

Andrikopoulos, A., \& Diakidis, N. (2007). Financial reporting practices on the internet: the case of companies listed in the Cyprus Stock Exchange. SSRN, 1-22. https://papers.ssrn.com/sol3/papers.cfm?abstract_id=999183 
Andrikopoulos, A., Merika, A. A., Triantafyllou, A., \& Merikas, A. G. (2013). Internet disclosure and corporate performance: A case study of the international shipping industry. Transportation Research Part A: Policy and Practice, 47, 141-152. https://doi.org/10.1016/j.tra.2012.10.016

Aqel, S. (2014). Determinants of corporate reporting on the internet: An analysis of companies listed on the Istanbul Stock Exchange (ISE). Research Journal of Finance and Accounting, 5(8), 139-149. https://www.iiste.org

Arango, E., Ceballos, R., \& Osorio, A. (2020). Investigación en publicidad política\#: un análisis bibliométrico Political advertising research: A bibliometric analysis. Revista Guillermo de Ockham, 18(2), 181-189. https://doi.org/1 $0.21500 / 22563202.4574$

Aranguren, N., \& Maldonado, S. (2015). La divulación de información social en América Latina: el caso de México, Chile y Colombia. XV Congreso AECA. http://www.aeca1.org/pub/on_line/comunicaciones_xvcongresoaec a/cd/48h.pdf

Ariff, A. M., Bin-Ghanem, H. O., \& Hashim, H. A. (2018). Corporate ownership, internet penetration and internet financial reporting: Evidence from the gulf cooperation council countries. Asian Journal of Business and Accounting, 11(1), 185-228. https://doi.org/10.22452/ajba.vol11nol.7

Ashbaugh, H., Johnstone, K. M., \& Warfield, T. D. (1999). Corporate reporting on the internet. Accounting Horizons, 13(3), 241-257. https://doi.org/10.2308/acch.1999.13.3.241

Bananuka, J. (2019). Intellectual capital, isomorphic forces and internet financial reporting: evidence from Uganda's financial services firms. Journal of Economic and Administrative Sciences, 36(2), 111-134. https://doi.org/10.1 108/jeas-03-2018-0042

Bonsón, E., \& Escobar, T. (2004). Voluntary, disclose of financial reporting on internet. A comparative world-wide analysis. Revista Espanola de Financiacion y Contabilidad, 33(123), 1063-1101. https://doi.org/10.1080/021 02412.2004.10779539

Bowrin, A. R. (2015). Comprehensiveness of internet reporting by Caribbean companies. Journal of Accounting in Emerging Economies, 5(1), 2-34. https://doi.org/10.1108/jaee-08-2011-0028

Caba, C., Rodríguez, M. P., \& López, A. M. (2008). E-Government process and incentives for online public financial information. Online Information Review, 32(3), 379-400. https://doi.org/10.1108/14684520810889682

Cárcaba, A., \& García, J. (2010). Determinants of online reporting of accounting information by Spanish local government authorities. Local Government Studies, 36(5), 679-695. https://doi.org/10.1080/03003930.2010 .506980

Cárcaba, A., \& García, J. (2008). Determinantes de la divulgación de información contable a través de Internet por parte de los gobiernos locales. Revista Espanola de Financiacion y Contabilidad, 37(137), 63-84. https://doi.o $\mathrm{rg} / 10.1080 / 02102412.2008 .10779639$

Debreceny, R., \& Gray, G. L. (1999). Financial reporting on the internet and the external audit. International Journal of Phytoremediation, 21(1), 335-350. https://doi.org/10.1080/096381899336078

Desoky, A. M. (2009). Company characteristicsas determinants ofinternet financial reporting in emergingmarkets: The caseofegypt. Research in Accounting in Emerging Economies (Vol. 9). Elsevier. https://doi.org/10.1108/S14793563(2009)0000009004

Dolinšek, T., \& Lutar-Skerbinjek, A. (2018). Voluntary disclosure of financial information on the internet by large companies in Slovenia. Kybernetes, 47(3), 458-473. https://doi.org/10.1108/K-08-2016-0220

Dolinšek, T., Tominc, P., \& Skerbinjek, A. (2014). The determinants of internet financial reporting in Slovenia. Online Information Review, 38(7), 842-860. https://doi.org/10.1108/OIR-02-2014-0025

Fisher, R. T., \& Naylor, S. T. (2016). Corporate reporting on the Internet and the expectations gap: New face of an old problem. Accounting and Business Research, 46(2), 196-220. https://doi.org/10.1080/00014788.2015.102 9866

Fuertes-Callén, Y., Cuellar-Fernández, B., \& Pelayo-Velázquez, M. (2014). Determinants of online corporate reporting in three Latin American markets: The role of web presence development. Online Information Review, 38(6), 806831. https://doi.org/10.1108/OIR-10-2013-0240 
Gandía, J. L. (2008). Determinants of web site information by Spanish city councils. Online Information Review, 32(1), 35-57. https://doi.org/10.1108/14684520810865976

Gómez-Villegas, M., \& Montesinos, V. (2014). Gobierno Electrónico y Transparecia financiera y presupuestal de los departamentos en Colombia. Revista Venezolana de Gerencia, 19(68), 670-698. https://www.redalyc.org/arti culo.oa?id=29032819004

Guillamón, M. D., Bastida, F., \& Benito, B. (2011). The determinants of local government's financial transparency. Local Government Studies, 37(4), 391-406. https://doi.org/10.1080/03003930.2011.588704

Haro de Rosario, A., Caba, M., \& Sánchez, M. (2012). Determinantes de la transparencia online del sector del capital riesgo: el caso de España. Cuadernos de Contabilidad, 13(32), 43-64. http://hdl.handle.net/10554/23594

Keliwon, K. B., Abdul, Z., \& Hassan, M. S. (2018). Internet Financial Reporting (IFR) Disclosure Position and Firm Value. Asian Journal of Accounting and Governance, 9, 111-122. https://doi.org/10.17576/ajag-2018-09-10

Kelton, A. S., \& Yang, Y. wen. (2008). The impact of corporate governance on Internet financial reporting. Journal of Accounting and Public Policy, 27(1), 62-87. https://doi.org/10.1016/j.jaccpubpol.2007.11.001

Khrifech, D., Khoufi, W., \& Ghorbel, A. (2018). Web based financial disclosure in European con-text: European transparency directive analysis. International Journal of Accounting and Economics Studies, 6(1), 11. https://do i.org/10.14419/ijaes.v6i1.8631

Leão, M. V., João, R., \& Castelló, E. T. (2018). Thirty Years of Studies on Transparency, Accountability, and Corruption in the Public Sector: The State of the Art and Opportunities for Future Research. Public Integrity, 20(5), 512533. https://doi.org/10.1080/10999922.2017.1416537

Lee, R. L., \& Joseph, R. C. (2013). An examination of web disclosure and organizational transparency. Computers in Human Behavior, 29(6), 2218-2224. https://doi.org/10.1016/j.chb.2013.05.017

Lev, B. (2018). The deteriorating usefulness of financial report information and how to reverse it. Accounting and Business Research, 48(5), 465-493. https://doi.org/10.1080/00014788.2018.1470138

Lodhia, S., \& Stone, G. (2017). Integrated Reporting in an Internet and Social Media Communication Environment: Conceptual Insights. Australian Accounting Review, 27(1), 17-33. https://doi.org/10.1111/auar.12143

Maldonado-García, S., Escobar-Váquiro, N., \& Marín Gaviria, M. (2012). Divulgación de información contablefinanciera en el sitio web de empresas cotizadas de Chile y México: un estudio exploratorio. Cuadernos de Contabilidad, 13(33), 395-431. https://dialnet.unirioja.es/servlet/articulo?codigo $=5488508$

Marston, C., \& Polei, A. (2004). Corporate reporting on the Internet by German companies. International Journal of Accounting Information Systems, 5(3), 285-311. https://doi.org/10.1016/j.accinf.2004.02.009

Mohd, N. A., \& Weetman, P. (2006). Perpetuating traditional influences: Voluntary disclosure in Malaysia following the economic crisis. Journal of International Accounting, Auditing and Taxation, 15(2), 226-248. https://doi. org/10.1016/j.intaccaudtax.2006.08.001

Mokhtar, E. S. (2017). Internet financial reporting determinants: a meta-analytic review. Journal of Financial Reporting and Accounting, 15(1), 116-154. https://doi.org/10.1108/jfra-07-2016-0061

Montesinos, V. (2009). Transparencia y responsabilidad en el sector público: el papel de la información financiera en tiempos de crisis. XV Congreso AECA, 26-28.

Moreno-Enguix, M. del R., Gras-Gil, E., \& Henández-Fernández, J. (2019). Relation between internet financial information disclosure and internal control in Spanish local governments. Aslib Journal in Information Management, 71(2), 176-194. https://doi.org/10.1108/AJIM-06-2018-0150

Nurunnabi, M., \& Hossain, M. A. (2012). The voluntary disclosure of internet financial reporting (IFR) in an emerging economy: A case of digital Bangladesh. Journal of Asia Business Studies, 6(1), 17-42. https://doi.org/10.1108 $/ 15587891211190688$

Omran, M. A., \& Ramdhony, D. (2016). Determinants of internet financial reporting in African markets: the case of Mauritius. Journal of Developing Areas, 50(4), 1-18. https://doi.org/10.1353/jda.2016.0150 
Pina, V., Torres, L., \& Royo, S. (2010). Is e-government promoting convergence towards more accountable local governments? International Public Management Journal, 13(4), 350-380. https://doi.org/10.1080/10967494 .2010 .524834

Pinto, I., \& Ng Picoto, W. (2016). Configurational analysis of firms' performance: Understanding the role of Internet financial reporting. Journal of Business Research, 69(11), 5360-5365. https://doi.org/10.1016/j.jbusres.2016.0 4.138

Pivac, S., Vuko, T., \& Cular, M. (2017). Analysis of annual report disclosure quality for listed companies in transition countries. Economic Research-Ekonomska Istrazivanja, 30(1), 721-731. https://doi.org/10.1080/1331677X.2 017.1311231

Qasim, A., \& Al Barghouthi, S. (2017). Corporate internet financial reporting practices of companies operating in emerging economies: An empirical investigation on listed companies in the United Arab Emirates. International Journal of Business and Society, 18(Special Issue 3), 641-649. https://www.researchgate.net/publication/3231 86355

Rivera-Arrubla, Y. A., Zorio-Grima, A., \& García-Benau, M. A. (2017). Integrated reports: Disclosure level and explanatory factors. Social Responsibility Journal, 13(1), 155-176. https://doi.org/10.1108/SRJ-02-2016-0033

Rodríguez, M. P., Alcaide, L., \& López, A. M. (2013). Determinants of Financial Transparency in Government. International Public Management Journal, 16(4), 557-602. https://doi.org/10.1080/10967494.2013.849169

Sandhu, A., \& Singh, B. (2019). Board composition and corporate reporting on internet: Indian evidence. Journal of Financial Reporting and Accounting, 17(2), 292-319. https://doi.org/10.1108/JFRA-05-2017-0031

Serrano-Cinca, C., Fuertes-Callén, Y., \& Gutiérrez-Nieto, B. (2007). Online reporting by banks: A structural modelling approach. Online Information Review, 31(3), 310-332. https://doi.org/10.1108/14684520710764 096

Sharma, N. (2013). Theoretical Framework for Corporate Disclosure Research. Asian Journal of Finance \& Accounting, 5(1). https://doi.org/10.5296/ajfa.v5i1.3210

Sia, C. J., Brahmana, R., \& Memarista, G. (2018). Corporate internet reporting and firm performance: Evidence from Malaysia. Contemporary Economics, 12(2), 153-164. https://doi.org/10.5709/ce.1897-9254.269

Siddaway, A. P., Wood, A. M., \& Hedges, L. V. (2019). How to Do a Systematic Review: A Best Practice Guide for Conducting and Reporting Narrative Reviews, Meta-Analyses, and Meta-Syntheses. Annual Review of Psychology, 70(1), 747-770. https://doi.org/10.1146/annurev-psych-010418-102803

Sriram, R. S., \& Laksmana, I. (2006). Corporate Web site reports: Some evidence on relevance and usefulness. Information Resources Management Journal, 19(3), 1-17. https://doi.org/10.4018/irmj.2006070101

Stechemesser, K., \& Guenther, E. (2012). Carbon accounting: A systematic literature review. Journal of Cleaner Production, 36, 17-38. https://doi.org/10.1016/j.jclepro.2012.02.021

Twizeyimana, J. D., \& Andersson, A. (2019). The public value of E-Government - A literature review. Government Information Quarterly, 36(2), 167-178. https://doi.org/10.1016/j.giq.2019.01.001

Van, N., \& Waltman, L. (2010). Software survey\#: VOSviewer , a computer program for bibliometric mapping. Scientometrics, 84, 523-538. https://doi.org/10.1007/s11192-009-0146-3

Xiang, Y., \& Birt, J. L. (2021). Internet reporting, social media strategy and firm characteristics - an Australian study. Accounting Research Journal, 34(1), 43-75. https://doi.org/10.1108/ARJ-09-2018-0154

Xiao, J. Z., Yang, H., \& Chow, C. W. (2004). The determinants and characteristics of voluntary Internet-based disclosures by listed Chinese companies. Journal of Accounting and Public Policy, 23(3), 191-225. https://doi. org/10.1016/j.jaccpubpol.2004.04.002

Yassin, M. M. (2017). The determinants of internet financial reporting in Jordan: Financial versus corporate governance. International Journal of Business Information Systems, 25(4), 526-556. https://doi.org/10.1504/I JBIS.2017.085176

Yusuf, I. (2013). Internet corporate financial reporting - A study of quoted Nigerian companies. African J. of Accounting, Auditing and Finance, 2(3), 233. https://doi.org/10.1504/ajaaf.2013.057631 


\section{Notas}

* Artículo de investigación

1 Electronic online reporting, internet accounting, internet disclosure, internet financial disclosure, internet financial reporting, internet for financial reporting, online reporting, online financial reporting, web reporting, web based accounting, web financial reporting.

\section{Licencia Creative Commons CC BY 4.0}

Sugerencia de citación: Rincón-Soto, C., Gómez-Villegas, M., \& Rivera-Arrubla, Y. (2021). Los diseños de índices de divulgación de información financiera en Internet. Una revisión sistemática de la literatura. Cuadernos de Administración, 34. https://doi.org/10.11144/Javeriana.cao34.didifi 